\title{
Diagnostics of Polymer Composite Materials by Waveguide Method
}

\author{
German V. Dmitrienko ${ }^{1, a^{*}}$, Dmitry V. Mukhin ${ }^{1}$ and Aleksandr A. Fedorov ${ }^{1}$ \\ ${ }^{1}$ Ulyanovsk Technical University. Institute of Aviation Technologies and Management, 13A, \\ Sozidateley Avenue, Ulyanovsk, Russia, 432072

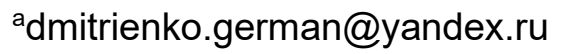

\section{Keywords: Polymer Composite Materials, Carbon Plastics, Dielectric Permittivity}

\begin{abstract}
This article considers the use of radio wave method for testing polymer composites used as structural material for manufacture of aircrafts. Measuring process, analysis and calculation by integral equation method are described.
\end{abstract}

\section{Introduction}

Since the middle of the last century polymer composite materials are gaining widespread use and occupy an increasingly wide scope of application across the full range of technology. The most important feature of these materials is that their properties depend not only on the properties of the components they are composed of, but also on the structure of the material being created, as well as on the technology of manufacturing parts from them. This leads to the steady growth of the range of new PCMs in order to obtain the properties most desirable for use in new areas. This feature also has a downside: any violations of the production process when manufacturing parts from PCM can lead to an undesirable change in the characteristics of future parts. In order to prevent this, the process of manufacturing parts from PCM includes various methods of nondestructive testing (NDT), which allow to control the parameters of the production process of PCM parts manufacturing, to adjust the process itself to eliminate arising defects and to reject defect parts at an early stage of manufacturing, if the revealed defect does not seem possible to be eliminated. The most informative NDT methods are radio wave methods with the information parameter being the complex reflection coefficient or the complex transmission coefficient of an electromagnetic wave, depending on the structure of the polymer material.

The complex dielectric constant $\underline{\varepsilon}$ of the PCM is measured by the wave guide technique according to the method and the measuring scheme described in [1-3]. A sample of the material to be measured is placed on the open end of the waveguide as a short-circuit, and the values $\underline{\varepsilon}$ and $\tan \delta$ are calculated using the Fresnel formulas from the measured complex reflection coefficient.

The complex reflection coefficient has the form

$$
\underline{R}=\frac{1-\sqrt{\underline{\varepsilon}}}{1+\sqrt{\varepsilon}} \text {, where } \underline{R} \text { is the complex reflection coefficient, } \underline{\varepsilon}=\varepsilon(1-j \tan \delta),
$$

from the formula, the values of the dielectric constant $-\varepsilon$ and the tangent of the angle of dielectric losses - $\tan \delta$ are expressed:

$$
\varepsilon=\frac{\left(1-R^{2}\right)^{2}-4 R^{2} \sin ^{2} \phi}{\left(1+R^{2}+2 R \cos \phi\right)^{2}}, \quad \tan \delta=\frac{4 R\left(1-R^{2}\right) \sin \phi}{\left(1-R^{2}\right)^{2}-4 R^{2} \sin ^{2} \phi}
$$


a

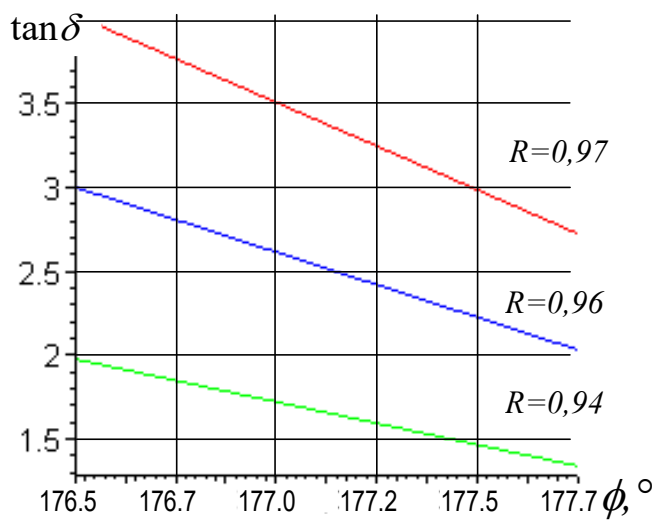

$\mathrm{b}$

Fig. 1. Dependences of $\varepsilon$ and $\tan \delta$ on the modulus $|R|$ and the phase $\phi$ of the reflection coefficient

According to the formulas (Eq.1), the dependences of the relative permittivity $\varepsilon$ and the tangent of the dielectric loss angle $\tan \delta$ on the modulus of the reflection coefficient and the phase of the reflection coefficient $(|R|, \phi)$ are plotted, as shown in Fig. 1. It is seen from the analysis of the dependences (Fig. 1) that the phase of the reflection coefficient tends to the phase of total reflection $\phi \rightarrow 180^{\circ}$; the graphs ascend very rapidly, which means that even a slight instrument error in measuring the phase $\phi$ will lead to a large final error in determining the relative permittivity $\varepsilon$.

In order to improve the accuracy of calculating and processing the obtained measurement results, a more rigorous method from an electrodynamic point of view is applied - that is the method of integral equations (IEM). The procedure for deriving integral equations, specified in [1$3]$, is realized by studying the surface currents induced on the surface of the PCM sample and the metal structure of the waveguide sensor by a probing electromagnetic wave.

The design of the measuring sensor is determined by the capabilities of the IEM [4-8], which allows you to numerically calculate the complex reflection coefficient in case of a sensor created on the basis of a round waveguide with a conical horn (Fig. 2). It is possible to measure the dielectric parameters of electrically thin PCM samples (conductive carbon plastics with a thickness of 1-2 mm, or fiberglass used as protective caps for the radar of an aircraft) by transmitting electromagnetic wave through the material with a receiving antenna added at the output of the measuring sensor.

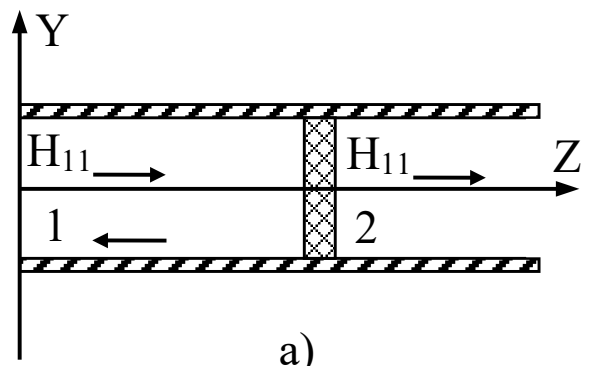

a)

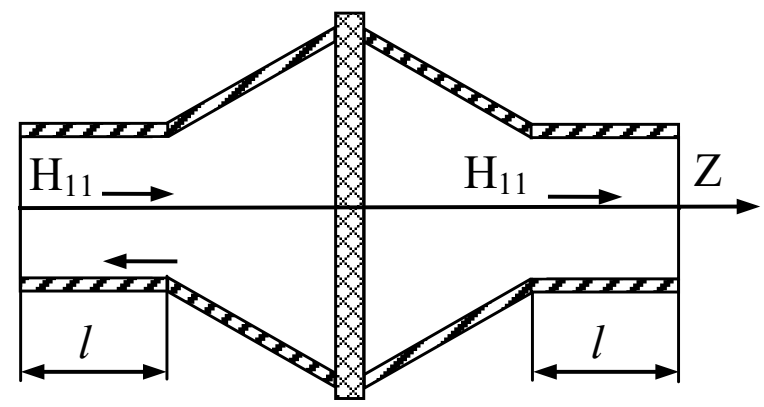

б)

Fig. 2. Waveguide Transducer Design for Transmission Measurements:

a) - on a round waveguide; b) - on a round waveguide with a conical horn. 
To measure the PCM dielectric characteristics for the transmitting an electromagnetic wave through the material, an emitter with an increased power is used to facilitate such an intensity of radiation at the output from the sensor, sufficient for measuring instruments. The reason for the increase in the measurement power is caused by high attenuation of the electromagnetic wave in the PCM.

When measuring by the transmitting electromagnetic wave, the measured PCM sample is represented as an electrically thin body. The measured parameter is the complex transmission coefficient $[9,10]$.

A system of integral equations for the outer (illuminated) and rear (shaded) boundaries of a thin body is compiled using the equations. In integral equations the described current on the illuminated surface is represented as $J^{M}=J_{n a d}^{M}+J_{\text {omp }}^{M}$, and on the shaded surface as $J^{M}=J_{n p o}^{M}$. On the outer surface (L1) the probing wave field is created by the current, on the rear surface (L2) the transmitted wave will be described by the induced currents $J_{2}^{M}$ and $J_{2}^{E}$. Integral equations are derived in the same manner as in $[9,10]$, taking into account the boundary conditions $[n, E]=Z[n,[n, H]]$ at the boundaries $L 1$ and $L 2$, where $n$ is the outward normal directed into the PCM sample, $Z$ is the diagonal matrix of the surface impedance $Z=\left|\begin{array}{cc}Z_{11} & 0 \\ 0 & Z_{22}\end{array}\right|$. The matrix character of the impedance shows that it can be different in different directions, in case of PCM anisotropy. The system of equations has the form:

$$
\begin{aligned}
& -\frac{1}{2} \widetilde{J}_{1}^{M}\left(p_{1}\right)+\int_{L 1} \xi_{1}^{M} \widetilde{J}_{1}^{M}\left(q_{1}\right) d l+\int_{L 2} \xi_{1}^{E} J_{1}^{E}\left(q_{1}\right)-\xi_{1}^{M} \widetilde{Z}\left[\vec{n}\left(q_{1}\right), J_{1}^{E}\left(q_{1}\right)\right] d l=0 \\
& \frac{1}{2} J_{2}^{M}\left(p_{2}\right)+\int_{L 1} \xi_{1}^{M} \widetilde{J}_{1}^{M}\left(q_{2}\right) d l+\int_{L 2}\left(\xi_{2}^{E}-\xi_{1}^{E}\right) J_{2}^{E}\left(q_{2}\right)-\left(\xi_{2}^{M}-\xi_{1}^{M}\right) \widetilde{Z}\left[\vec{n}\left(q_{2}\right), J_{2}^{E}\left(q_{2}\right)\right] d l=0 \\
& \frac{1}{2} J_{2}^{E}\left(p_{2}\right)+\int_{L 1} h_{1}^{M} \widetilde{J}_{1}^{M}\left(q_{2}\right) d l+\int_{L 2}\left(h_{2}^{M}-h_{1}^{M}\right) J_{2}^{E}\left(q_{2}\right)-\left(h_{2}^{M}-h_{1}^{M}\right) \widetilde{Z}\left[\vec{n}\left(q_{2}\right), J_{2}^{E}\left(q_{2}\right)\right] d l=0
\end{aligned}
$$

where $p_{1}, q_{1}$ are points on the boundary $L 1$, and a $p_{2} q_{2}$ on the boundary $L 2$.

Equation (Eq.2) for the outer surface L1 is similar to the equations for a homogeneous semiinfinite body. The last integral term on the left side of it considers the reflection from the rear surface. Equations (Eq.3) and (Eq.4) describe the field of the transmitted wave, with the first integral terms considering the influence of the outer surface. The boundary conditions $\widetilde{J}^{M}=-\widetilde{Z}\left[n, J^{E}\right], \widetilde{Z}=Z / \sqrt{\mu_{0} / \varepsilon_{0}}$ are satisfied on both surfaces. The resulting equations are Fredholm equations of the second kind.

Integral equations are solved by a numerical method (Galerkin's method) [4,11-16].

The PCM sample was tested according to the proposed theory. Based on the measurement results, the value of the relative dielectric constant $\varepsilon=940 \pm 30 \%$ and the tangent of the dielectric loss angle $\tan \delta=1,3 \pm 40 \%$, with a reflection coefficient modulus being 0.94 , were determined.

\section{References}

[1] A. A. Brandt, Investigations of Dielectrics at Ultrahigh Frequencies [in Russian], Moscow: GIFML (1963) p.404

[2] G.V. Belov, Technical quality control of products made of carbon materials [in Russian], Moscow: Izdatel'stvo standartov (1991) p.92. 
[3] G.V. Dmitrienko Method for measuring the complex reflection coefficient of low-impedance composite materials in the microwave range using intermediate standards [In Russian], Metrologia (2008) No 4. pp. 31-38.

[4] E.N. Vasiliev Excitation of bodies of revolution [In Russian] Moscow, Radio and communication (1987) p. 271.

[5] M. Zh. Kislyuk, Method of Secondary Waves in Problems of Electrodynamics [in Russian], LÉTI, Leningrad (1970) p.151.

[6] G.V. Dmitrienko, A.A. Fedorov and G.L. Rivin, Radio-Frequency Method for Diagnostics of Aeronautical Polymer Composite For Newly Launched Products [in Russian] FGUP „VIAM“ (2018) ISBN 978-5-905217-30-2. https://conf.viam.ru/conf/284/proceedings

[7] C.A. Fletcher, Computational galerkin methods [in Russian] Izdatel'stvo Mir, (1988) p. 347

[8] V.A. Dandarov, Numerical solution of the 2-dimensional integral Fredholm equation in the problem of radio wave propagation over an irregular earth surface [in Russian] Radiotekhnika $i$ elektronika (2002) Vol.47, No.11, pp.1329-1334

[9] G.V. Dmitrienko, V.G. Anisimov, Measurement of the dielectric constant of low-impedance composite materials at microwave frequencies [in Russian] Izmeritel'naya tekhnika (2009) No. 2. https://doi.org/10.1007/s11018-009-9236-0

[10] G.V. Dmitrienko, N.A. Trefilov, RF Patent No 2194285 IPC $^{7}$ G01 R 27/04. A method for determining large values of the complex dielectric constant of impedance materials, applied for 13.03.2001; published on 10.12.2002, Bul No.34.

[11] G.V. Dmitrienko, L.V. Fedotov, Quality control process of low-impedance composite materials at microwave frequency [in Russian] Kachestvo, innovatsii, obrazovanie (2009) No.10 [12] G.D Malushkov, Methods of solving problems of the electromagnetic excitation of bodies of revolution (January 1975) · Izvestiya Vysshikh Uchebnykh Zavedenij.

https://doi.org/10.1007/BF01037102

[13] A. F. Verlan and V. S. Sizikov, Integral Equations: Methods, Algorithms, Programs [in Russian], Naukova Dumka, Kyiv (1986) p.544

[14] N. A. Khizhnyak, Integral Equations of Macroscopic Electrodynamics [in Russian], Naukova Dumka, Kiev (1986) p.256

[15] VV Nikol'sky, Projection methods in electrodynamics [in Russian] Prikladnaya elektrodinamika (1977)

[16] P.V. Novitsky and I.A. Zograf, Evaluation the Errors of Measurement Results, Moscow, Energoatomizdat Publishing (1985) p.247. 